\title{
LATTICE-ORDERED POWER SERIES FIELDS
}

\author{
R. H. REDFIELD
}

(Received 2 April 1990)

Communicated by T. E. Hall

\begin{abstract}
A lattice-ordered power series algebra of a totally ordered field over a rooted abelian group may be constructed in a way that is arbitrary only in requiring that a factor set be chosen in the field and an extended total order be chosen on the group modulo its torsion subgroup. The resulting algebra is a field if and only if the subalgebra of elements with torsion support form a field. It follows that if the torsion subgroup may be independently embedded in the algebraic closure of the totally ordered field, or if the resulting algebra has no zero-divisors, then the algebra is a field. The set of supporting subsets for the power series may be characterized abstractly in such a way that previous representation theorems of lattice-ordered fields into power series algebras may be applied to produce representations into power series fields.

1991 Mathematics subject classification (Amer. Math. Soc.): primary 06 F 25; secondary 06 F $15,12 \mathrm{~J} 15,13 \mathrm{~J} 05,16$ A 27,16 A 86.

Keywords and phrases: Lattice-ordered fields, lattice-ordered algebras, power series, convolution, Hahn's Theorem, representations of ordered algebraic structures, products of ordered algebraic structures.
\end{abstract}

Typically, the objects which are used to represent commutative ordered algebraic structures are ordered products of totally ordered structures of the same type. The totally ordered Hahn groups (cf. [8, page 59], [9]) which represent totally ordered abelian groups, the lattice-ordered Hahn groups (cf.

(C) 1992 Australian Mathematical Society $0263-6115 / 92 \$ A 2.00+0.00$

This paper was written during a leave at Monash University. I would like to thank Hamilton College for granting me the leave and Monash for having me as a visitor. I would especially like to thank Professor J. B. Miller for his hospitality and Professor T. E. Hall for his help in proving the original version of Lemma 1.3. Professor Stuart Steinberg subsequently pointed out an error in the proof of Proposition 1.4. Correcting this error resulted in the present completely different version of the lemma. 
[7]) which represent lattice-ordered abelian groups, the Lorenzen representations (cf. [1, page 26], [3], [12]) of lattice-ordered abelian groups as subdirect cardinally ordered products of totally ordered abelian groups, and the totally ordered power series fields (cf. [6], [11], [13]) which represent totally ordered fields are all examples of such objects. In the noncardinally ordered cases, the possible supporting sets are restricted in such a way that the appropriate order may be defined on the remaining elements of the product. With these examples in mind, a general process for constructing lattice-ordered power series rings was described in [16], [17] and [18] (cf. [4], [20], [21] and [22] as well) and was used for representing certain lattice-ordered fields in [19].

The representations described in [19] embed certain lattice-ordered fields in power series algebras of totally ordered fields over rooted abelian groups. Obviously the best such representations would embed the fields in power series fields rather than in power series algebras. The object of this paper is twofold, first to describe a way of constructing lattice-ordered power series fields which is less arbitrary than the general method described in [16] and [17] (and very much a generalization of the concrete examples given in [18]) and second to use this construction to sharpen the representation theorems of [19].

The paper progresses as follows. The first section lays the groundwork for the construction by abstractly describing the subsets of the index group which will support the elements of the power series algebras. The second section describes the general construction of these algebras. The special case of this construction which is of primary interest here is arbitrary only in requiring that a factor set be chosen in the totally ordered field and an extended total order be chosen on the index group modulo its torsion subgroup. Once these choices have been made, the supporting sets are precisely those which have only finitely many elements in any coset of the torsion subgroup and, modulo this subgroup, are inversely well-ordered with respect to the chosen total order. The third section characterizes those instances in which these special power series algebras are fields. Internally the subalgebra of elements with torsion support must be a field; externally the factor set must arise from an independent embedding of the torsion subgroup into the algebraic closure of the totally ordered field. Since each of these special power series algebras comes equipped with a canonical lattice order, these results determine exactly when a canonically lattice-ordered power series algebra is in fact a lattice-ordered field. The fourth section contains two representation theorems derived from those in [19]. The first is a general one and the second gives three equivalent statements, two of which form the analogue for lattice-ordered fields of the usual characterization in the theory of lattice-ordered groups of cardinal sums of totally ordered groups. Specifically, lattice-ordered fields which are 
isomorphic to power series fields all of whose elements have finite support are characterized as those lattice-ordered fields in which every positive element exceeds at most a finite number of disjoint elements, no convex totally ordered subgroup is bounded, and all the (order) basic elements have positive multiplicative inverses.

For the basic theory of lattice-ordered algebraic structures, we refer the reader to the books by Fuchs [8], Conrad [5], Bigard, Keimel and Wolfenstein [2], and Anderson and Feil [1].

We will use the following notation and terminology without further comment. If $G$ is a partially ordered group, then $G^{+}=\{g \in G \mid g>0\}$ and $G^{*}=\{g \in G \mid g \neq 0\}$. A lattice subalgebra of a lattice-ordered algebra is a subset which both is a subalgebra and a sublattice. We use $\mathbb{N}$ to denote the natural numbers $1,2, \ldots$. All algebras are associative and have unit element 1 , with subalgebras having the same unit element. Homomorphisms between algebras preserve unit elements. All fields are commutative.

For use in the sequel, we record the following result from [8].

LemMA 0.1. Suppose that $(\Gamma, \cdot, \geq)$ is totally ordered abelian group and that $A$ is an inversely well-ordered subset of $(\Gamma, \geq)$ such that $l \geq \alpha$ for all $\alpha \in A$. Then $\bigcup_{n=1}^{\infty} A^{n}$ is also an inversely well-ordered subset of $(\Gamma, \geq)$ and for each $\gamma \in \Gamma$, there are only finitely many positive integers $n$ such that $\gamma \in A^{n}$.

Proof. This result is exactly what Fuchs proved to prove the lemma on page 135 of [8].

\section{Strongly supporting sets}

In general, lattice-ordered power series algebras may be constructed from totally ordered fields and supporting collections of subsets of rooted groups (see [16], [17] and [18]). In Section 2 below, we will see that supporting collections which satisfy an additional condition-the strongly supporting collections-ensure that the resulting algebra is contained in an algebra which is defined arbitrarily and which, as we will see in Section 3 below, is frequently a field. In this section, we define and investigate strongly supporting collections.

Recall first that a partially ordered group $(\Delta, \cdot \geq)$ is rooted if for all $\delta \in \Delta$, $[\delta, \infty)$ is totally ordered and that a subset $\Gamma \subseteq \Delta$ is locally inversely wellordered if for all $\gamma \in \Gamma$, the set $\{\delta \in \Gamma \mid \delta \geq \gamma\}$ is inversely well-ordered. Clearly the partially ordered set $\left(2^{\Delta}, \subseteq\right)$ is a partially ordered semigroup 
with the respect to the multiplication $A B=\{\alpha \beta \mid \alpha \in A, \beta \in B\}$. A subset $X$ of $2^{\Delta}$ is than a supporting subset if it satisfies the following conditions, where $l$ denotes the identity of $\Delta$ :

$\left(P_{0}\right)$ every element of $X$ is locally inversely well-ordered;

$\left(P_{1}\right) \quad X$ contains all the atoms of $\left(2^{\Delta}, \subseteq\right)$;

$\left(P_{2}\right) \quad X$ is an ideal of the lattice $\left(2^{\Delta}, \subseteq\right)$;

$\left(P_{3}\right) \quad X$ is a subsemigroup of $\left(2^{\Delta}, \cdot\right)$;

$\left(P_{4}\right) \quad X$ if $A, B \in X$, then $\{(\alpha, \beta) \in A \times B \mid \alpha \beta=\imath\}$ is finite.

For a set $\Delta$ and a set of subsets $X \subseteq 2^{\Delta}$, let $\operatorname{Cou}(X)$ denote the set of all countable subsets of elements of $X$ (where "countable" means finite or countably infinite). A supporting subset $X$ is then strongly supporting if it satisfies the additional condition:

$\left(P_{5}\right)$ if $\varnothing \neq A \in \operatorname{Cou}(X)$, there exists $\mu \in A$ such that $\bigcup_{n=1}^{\infty}\left(\mu^{-1} A\right)^{n} \in X$.

Strongly supporting subsets are abundant. To see this, see $I W(\geq)$ denote the set of inversely well-ordered subsets of any totally ordered set $(\Delta, \geq)$.

Proposition 1.1. If $(\Delta, \cdot \geq)$ is a totally ordered abelian group, then $I W(\geq)$ is a strongly supporting subset of $2^{\Delta}$.

Proof. It was shown in [18] that $I W(\geq)$ is supporting, and by Lemma 0.1 , if $\varnothing \neq A \in I W(\geq)$ and $\mu$ is the maximal element of $A$, then $\bigcup_{n=1}^{\infty}\left(\mu^{-1} A\right)^{n} \in I W(\geq)$. The weaker condition $\left(P_{5}\right)$ follows immediately.

The next two results describe how to construct strongly supporting subsets for rooted abelian groups which may not be totally ordered.

First recall that if $\Delta_{t}$ denotes the set of all elements of the abelian group $\Delta$ which are of finite order, then it is well known [23, page 176] that $\Delta_{t}$ is a subgroup of $\Delta$ for which the quotient group $\Delta / \Delta_{t}$ is torsion-free. If $(\Delta, \cdot, \geq)$ is a partially ordered abelian group with convex subgroup $\Gamma$, we may define a binary relation $\geq$ on $\Delta / \Gamma$ by letting

$$
\begin{gathered}
\alpha \Gamma \geq \Gamma \quad \text { if and only if there exists } \delta \in \Delta \text { such that } \alpha \delta \geq \imath ; \\
\alpha \Gamma \geq \gamma \Gamma \quad \text { if and only if } \alpha \gamma^{-1} \Gamma \geq \Gamma .
\end{gathered}
$$

If $\Gamma$ is directed, then it is also well known [8, page 18] that $(\Delta / \Gamma, \cdot, \geq)$ is a partially ordered group. In the case of rooted groups, we also have the following. 
Proposition 1.2. If $(\Delta, \cdot, \geq)$ is a rooted abelian group, then the quotient group $\left(\Delta / \Delta_{t}, \cdot, \geq\right)$ is a torsion-free rooted abelian group.

Proof. If $\eta \Delta_{t} \geq \Delta_{t}$, and $\gamma \Delta_{t} \geq \Delta_{t}$, then it is easy to see that $\eta \gamma \Delta_{t} \geq \Delta_{t}$. If $\eta \Delta_{t} \geq \Delta_{t}$, then $\eta \tau \geq \imath$ for some $\tau \in \Delta_{t}$. Since $\tau \in \Delta_{t}$, there exists $n \in \mathbb{N}$ such that $\tau^{n}=\imath$, and hence $l \leq \eta^{n} \tau^{n}=\eta^{n}$. Similarly, if $\Delta_{t} \geq \eta \Delta_{t}$ as well, then there exists $m \in \mathbb{N}$ such that $\imath \leq \eta^{-m}$. Then $\left(\eta^{m n}\right)^{-1} \leq \imath \leq \eta^{m n}$. But by [19, Proposition 8.2], the set of all elements of $\Delta$ which are comparable to $l$ is a totally ordered subgroup of $\Delta$; hence $\eta^{m n}=t$. Then $\eta \in \Delta_{t}$ and hence it follows from [8, page 13 , Theorem 2] that $\left(\Delta / \Delta_{t}, \cdot, \geq\right)$ is a partially ordered group. To see that it is rooted, suppose that $\eta \Delta_{t} \leq \alpha \Delta_{t}, \beta \Delta_{t}$. Then, for some $\sigma, \tau \in \Delta_{t}, l \leq \alpha \eta^{-1} \tau$ and $l \leq \beta \eta^{-1} \sigma$. Since $[l, \infty)$ is totally ordered, we may assume without loss of generality that $\alpha \eta^{-1} \tau \leq \beta \eta^{-1} \sigma$. Then $l \leq \beta \alpha^{-1} \sigma \tau^{-1}$ and hence $\Delta_{t} \leq \beta \alpha^{-1} \Delta_{t}$, that is, $\alpha \Delta_{t} \leq \beta \Delta_{t}$. It follows that $\left(\Delta / \Delta_{t}, \cdot, \geq\right)$ is a rooted abelian group. As was noted above, it is well known that $\left(\Delta / \Delta_{t}, \cdot\right)$ is torsion-free.

A partial order $\geq$ on a set $\Delta$ extends a partial order $\geq$ on $\Delta$ if $\alpha \geq \beta$ whenever $\alpha \geq \beta$; if $(\Delta, \cdot, \geq)$ is a partially ordered group, then we say that $\geq$ is an extended total order on $\Delta$ if $\geq$ extends $\geq$ and $\left(\Delta / \Delta_{t}, \cdot, \geq\right)$ is a totally ordered group. For any rooted abelian group $(\Delta, \cdot, \geq)$, by Proposition $1.2,\left(\Delta / \Delta_{t}, \cdot, \geq\right)$ is a torsion-free rooted abelian group, and hence by [5, page 0.4$]\left(\Delta / \Delta_{t}, \cdot, \geq\right)$ always has at least one extended total order. With these observations in mind, we will describe in Proposition 1.4 below how to construct strongly supporting subset for any rooted abelian group.

To prove Proposition 1.4, we need the following lemma. For it and Proposition 1.4, we need the following notation: if $(\Delta, \cdot, \geq)$ is a rooted abelian group and $A \in 2^{\Delta}$, then $A / \Delta_{t}=\left\{\alpha \Delta_{t} \mid \alpha \in A\right\}$.

LEMMA 1.3. Suppose that $(\Delta, \cdot, \geq)$ is a rooted abelian group and that $\geq$ is an extended total order on $\left(\Delta / \Delta_{t}, \cdot, \geq\right)$. Suppose further that $B \subseteq \Delta$ is such that $B / \Delta_{t} \in I W(\geq)$ and $\Delta_{t} \geq \beta \Delta_{t}$ for all $\beta \in B$. If $B^{n} \cap \delta \Delta_{t}$ is finite for all positive integers $n$ and all $\delta \in \Delta$, then $\left[\cup_{n=1}^{\infty} B^{n}\right] \cap \delta \Delta_{t}$ is finite for all $\delta \in \Delta$.

Proof. By Lemma 0.1, $\left[\cup_{n=1}^{\infty}\left(B / \Delta_{t}\right)^{n}\right] \in I W(\geq)$ and for all $\delta \in \Delta$, there are only finitely many $n$ such that $\delta \Delta_{t} \in\left(B / \Delta_{t}\right)^{n}$. By way of contradiction, suppose that there exists $\gamma \in \Delta$ such that $\left[\bigcup_{n=1}^{\infty}\right] \cap \gamma \Delta_{t}$ is infinite. By hypothesis, $B^{n} \cap \gamma \Delta_{t}$ is finite for all $n$, and hence there exist positive integers $n[1]<n[2]<\cdots$ and elements $\eta_{n[i]} \in \gamma \Delta_{\imath}$ such that for all $i, \eta_{n[i]} \in B^{n[i]}$. 
Then for infinitely many $n[i], \gamma \Delta_{t}=\eta_{n[i]} \Delta_{t} \in\left(B / \Delta_{t}\right)^{n[i]}$. This is a contradiction and hence $\left[\bigcup_{n=1}^{\infty} B^{n}\right] \cap \delta \Delta_{t}$ must be finite for all $\delta \in \Delta$.

Proposition 1.4. Suppose that $(\Delta, \cdot, \geq)$ is a rooted abelian group and that $\geq$ is an extended total order on $\left(\Delta / \Delta_{t}, \cdot, \geq\right)$. If $X(\geq)$ is the collection of subsets $A \subseteq \Delta$ such that $A / \Delta_{t} \in I W(\geq)$ and for all $\delta \in \Delta, A \cap \delta \Delta_{t}$ is finite, then $X(\geq)$ is a strongly supporting subset of $2^{\Delta}$.

Proof. Suppose first that $A \in X(\geq)$ but, by way of contradiction, that $A$ is not locally inversely well-ordered. Then there exists a strictly increasing sequence $a_{1}<a_{2}<a_{3}<\cdots$ in $A$. In $\Delta / \Delta_{t}$, this sequence gives rise to the increasing sequence $a_{1} \Delta_{t} \leq a_{2} \Delta_{t} \leq a_{2} \Delta_{t} \leq \cdots$ in $A / \Delta_{t}$. It was shown in [18] that every element of $I W(\geq)$ is a locally inversely well-ordered subset of $\left(\Delta / \Delta_{t}, \geq\right)$, and hence there must exist $n$ such that $a_{k} \Delta_{t}=a_{n} \Delta_{t}$ for all $k \geq n$. But then $A \cap a_{n} \Delta_{t}$ contains the infinite set $\left\{a_{n}, a_{n+1}, a_{n+2}, \ldots\right\}$, which contradicts our choice of $A \in X(\geq)$. We conclude that $\left(P_{0}\right)$ holds for $X(\geq)$.

Clearly $\left(P_{1}\right)$ holds, and since $(A \cup B) / \Delta_{t}=A / \Delta_{t} \cup B / \Delta_{t},\left(P_{2}\right)$ holds as well. To see that $\left(P_{3}\right)$ holds, suppose by way of contradiction that for some $\delta \in \Delta, A B \cap \delta \Delta_{t}$ contains an infinite subset $\left\{a_{1} b_{1}, a_{2} b_{2}, a_{3} b_{3}, \ldots\right\}$. Then for all $i, a_{i} \in b_{i}^{-1} \delta \Delta_{t}$. But, for all $i, A \cap b_{i}^{-1} \delta \Delta_{t}$, is finite, and hence (by choosing a subsequence if necessary) we may assume for all $i \neq j$ that $b_{i}^{-1} \delta \Delta_{t} \neq b_{j}^{-1} \delta \Delta_{t}$, that is, that $b_{i} \Delta_{t} \neq b_{j} \Delta_{t}$. Since $B / \Delta_{t} \in I W(\geq)$, this in turn means that (again by choosing a subsequence if necessary) we may assume that $b_{1} \Delta_{t}>b_{2} \Delta_{t}>b_{3} \Delta_{t}>\cdots$. The same argument for the $a_{i}$ 's shows that we may assume that $a_{1} \Delta_{t}>a_{2} \Delta_{t}>a_{3} \Delta_{t}>\cdots$. But then $\delta \Delta_{t}=$ $a_{1} b_{1} \Delta_{t}>a_{2} b_{2} \Delta_{t}=\delta \Delta_{t}$, a contradiction, and we conclude that $\left(P_{3}\right)$ holds. For $\left(P_{4}\right)$, suppose that $A, B \in X(\geq)$ and observe that by Proposition 1.1, $\left\{\left(\alpha \Delta_{t}, \beta \Delta_{t}\right) \in A / \Delta_{t} \times B / \Delta_{t} \mid \alpha \beta \in \Delta_{t}\right\}$ is finite. However, for any $\alpha \in A$, there are only finitely many $\gamma \in A$ such that $\gamma \Delta_{t}=\alpha \Delta_{t}$ and similarly for $\beta \in B$, and hence $\left\{(\alpha, \beta) \in A \times B \mid \alpha \beta \in \Delta_{t}\right\}$ is finite. Since $l \in \Delta_{t}$, we conclude that $\left(P_{4}\right)$ holds.

To see that $\left(P_{5}\right)$ holds, suppose that $\varnothing \neq A \in \operatorname{Cou}(X(\geq))$ and let $\mu \in A$ be such that $\mu \Delta_{t}$ is maximal in $A / \Delta_{t}$. Then $\bigcup_{n=1}^{\infty}\left[\left(\mu \Delta_{t}\right)^{-1}\left(A / \Delta_{t}\right)\right]^{n} \in I W(\geq)$ by Lemma 0.1 . But $\left[\bigcup_{n=1}^{\infty}\left(\mu^{-1} A\right)^{n}\right] / \Delta_{t}=\bigcup_{n=1}^{\infty}\left[\left(\mu \Delta_{t}\right)^{-1}\left(A / \Delta_{t}\right)\right]^{n}$, and hence to show that $\bigcup_{n=1}^{\infty}\left(\mu^{-1} A\right)^{n} \in X(\geq)$, it suffices to show that $\left[\bigcup_{n=1}^{\infty}\left(\mu^{-1} A\right)^{N}\right] \cap$ $\delta \Delta_{t}$ is finite for all $\delta \in \Delta$. This follows from Lemma 1.3. For since $X(\geq)$ satisfies $\left(P_{1}\right)$ and $\left(P_{3}\right),\left(\mu^{-1} A\right)^{n} \in X(\geq)$ for all $n$ and hence $\mu^{-1} A / \Delta_{t} \in$ $I W(\geq)$ and $\left(\mu^{-1} A\right)^{n} \cap \delta \Delta_{t}$ is finite for all $\delta \in \Delta$. But clearly, since $\delta_{t}=$ $\mu^{-1} \mu \Delta_{t}, \Delta_{t} \geq \mu^{-1} \alpha \Delta_{t}$ for all $\alpha \in A$, and hence we may apply Lemma 1.3 to 
$\mu^{-1} A$ and conclude that $\left[\bigcup_{n=1}^{\infty}\left(\mu^{-1} A\right)^{n}\right] \cap \delta \Delta_{t}$ is finite for all $\delta \in \Delta$.

Having established in Proposition 1.4 that every rooted abelian group generates a strongly supporting collection, we now turn our attention to determining whether an arbitrary strongly supporting collection might be related to a collection of the form described in the proposition. We first note some basic facts about arbitrary strongly supporting collections (Propositions 1.5 and 1.7 and Corollaries 1.6 and 1.8) and then use them to prove (Proposition 1.9) that strongly supporting collections may be characterized as those supporting collections which are subcollections of strongly supporting collections of the form described in Proposition 1.4.

Proposition 1.5. If $(\Delta, \cdot, \geq)$ is a rooted abelian group, if $X$ is a strongly supporting subset of $2^{\Delta}$, and if $A \in X$, then $A$ has only finitely many elements of finite order.

Proof. Let $A_{t}$ denote the set of elements of $A$ which are of finite order, and by way of contradiction, suppose that $A_{t}$ has infinitely many members. Then $A_{t}$ has a countably infinite subset $C_{t}$. By $\left(P_{2}\right), C_{t} \in X$, and hence by $\left(P_{5}\right)$, there exists $\tau \in C_{t}$ such that $\bigcup_{n=1}^{\infty}\left(\tau^{-1} C_{t}\right)^{n} \in X$. Note that since $\tau \in C_{t}, \tau$ has finite order so that each element of $\tau^{-1} C_{t}$ also has finite order and hence has its inverse in $\bigcup_{n=1}^{\infty}\left(\tau^{-1} C_{t}\right)^{n}$. But since $C_{t}$ is infinite, $\tau^{-1} C_{t}$ is infinite, and thus

$$
\left\{(\alpha, \beta) \in \tau^{-1} C_{t} \times \bigcup_{n=1}^{\infty}\left(\tau^{-1} C_{t}\right)^{n} \mid \alpha \beta=\imath\right\}
$$

is also infinite. Since by $\left(P_{1}\right)$ and $\left(P_{3}\right), \tau^{-1} C_{t} \in X$, that the above set is infinite contradicts $\left(P_{4}\right)$.

The following stronger result follows immediately from Proposition 1.5.

Corollary 1.6. Suppose that $(\Delta, \cdot, \geq)$ is a rooted abelian group and that $X$ is a strongly supporting subset of $2^{\Delta}$. If $A \in X$ and $\eta \in \Delta$, then $A \cap \eta \Delta_{t}$ is finite.

Proof. By $\left(P_{1}\right)$ and $\left(P_{3}\right), \eta^{1} A=\left\{\eta^{-1}\right\} A \in X$, and thus, by Proposition 1.5, $\eta^{-1} A \cap \Delta_{t}$ is finite. It follows that $A \cap \eta \Delta_{t}$ is finite.

By Proposition 1.1, for a totally ordered abelian group $(\Delta, \cdot, \geq)$, the set $I W(\geq)$ of inversely well-ordered subsets of $(\Delta, \geq)$ is a supporting subset 
of $2^{\Delta}$. The next result and its corollary describe how a strongly supporting collection generates an extended total order on the quotient group $\Delta / \Delta_{t}$ in such a way that the elements of the strongly supporting collection, modulo $\Delta_{t}$, are all inversely well-ordered with respect to the extended total order.

We need the following additional notation for Proposition 1.7 and its corollary. For a rooted abelian group $(\Delta, \cdot, \geq)$ and a supporting subset $X \subseteq 2^{\Delta}$, we let $X / \Delta_{t}=\left\{A / \Delta_{t} \mid A \in X\right\}$.

Proposition 1.7. If $(\Delta, \cdot, \geq)$ is a rooted abelian group and $X$ is a strongly supporting subset of $2^{\Delta}$, then there exists an extended total order $\succcurlyeq$ on $\left(\Delta / \Delta_{t}, \cdot, \geq\right)$ such that $X / \Delta_{t} \subseteq I W(\succcurlyeq)$.

Proof. Define $\succcurlyeq$ on $\Delta / \Delta_{t}$ as follows:

$$
\begin{gathered}
\eta \Delta_{t} \succcurlyeq \Delta_{t} \text { if and only if }\left\{t, \eta^{-1}, \eta^{-2}, \eta^{-3}, \ldots\right\} \in X ; \\
\gamma \Delta_{t} \succcurlyeq \eta \Delta_{t} \text { if and only if } \gamma \eta^{-1} \Delta_{t} \succcurlyeq \Delta_{t} .
\end{gathered}
$$

To see that $\succcurlyeq$ is well-defined, we need to show that if $\left\{l, \eta^{-1}, \eta^{-2}, \eta^{-3}\right.$, $\ldots\} \in X$ and $\tau \in \Delta_{t}$, then $\left\{l,(\eta \tau)^{-1},(\eta \tau)^{-2},(\eta \tau)^{-3}, \ldots\right\} \in X$. If $\eta \in \Delta_{t}$, then $\left\{l,(\eta \tau)^{-1},(\eta \tau)^{-2},(\eta \tau)^{-3}, \cdots\right\}$ is finite and hence by $\left(P_{1}\right)$ and $\left(P_{2}\right)$, $\left\{l,(\eta \tau)^{-1},(\eta \tau)^{-2},(\eta \tau)^{-3}, \ldots\right\} \in X$. So suppose that $\eta \notin \Delta_{t}$. By $\left(P_{1}\right)$ and $\left(P_{2}\right),\{l, \eta \tau\} \in X$; by $\left(P_{5}\right)$, either $\left\{l,(\eta \tau)^{-1},(\eta \tau)^{-2},(\eta \tau)^{-3}, \ldots\right\} \in X$ or $\left\{l, \eta \tau,(\eta \tau)^{2},(\eta \tau)^{3}, \ldots\right\} \in X$. However, since $\tau$ has finite order and $\eta$ does not, $\left\{l, \eta \tau,(\eta \tau)^{2},(\eta \tau)^{3}, \ldots\right\}$ must contain an infinite subset of $\left\{l, \eta, \eta^{2}, \eta^{3}, \ldots\right\}$, and hence

$$
\left\{(\alpha, \beta) \in\left\{l, \eta \tau,(\eta \tau)^{2},(\eta \tau)^{3}, \ldots\right\} \times\left\{l, \eta^{-1}, \eta^{-2}, \eta^{-3}, \ldots\right\} \mid \alpha \beta=l\right\}
$$

must be infinite. It follows from $\left(P_{4}\right)$ that $\left\{l, \eta \tau,(\eta \tau)^{2},(\eta \tau)^{3}, \ldots\right\} \notin X$, and hence that $\left\{l,(\eta \tau)^{-1},(\eta \tau)^{-2},(\eta \tau)^{-3}, \ldots\right\} \in X$. We conclude that $\succcurlyeq$ is well-defined.

To see that $\left(\Delta / \Delta_{t}, \cdot, \succcurlyeq\right)$ is a totally ordered group, suppose first that $\eta \Delta_{t}, \gamma \Delta_{t} \geq \Delta_{t}$. Then $\left\{l, \eta^{-1}, \eta^{-2}, \eta^{-3}, \ldots\right\},\left\{l, \gamma^{-1}, \gamma^{-2}, \gamma^{-3}, \ldots\right\} \in X$. Since $\left\{l,(\eta \gamma)^{-1},(\eta \gamma)^{-2},(\eta \gamma)^{-3}, \ldots\right\} \subseteq\left\{l, \eta^{-1}, \eta^{-2}, \eta^{-3}, \ldots\right\}\left\{l, \gamma^{-1}\right.$, $\left.\gamma^{-2}, \gamma^{-3}, \ldots\right\},\left\{l,(\eta \gamma)^{-1},(\eta \gamma)^{-2},(\eta \gamma)^{-3}, \ldots\right\} \in X$ by $\left(P_{2}\right)$ and $\left(P_{3}\right)$ and thus $\eta \gamma \Delta_{t} \geq \Delta_{t}$. If $\eta \Delta_{t} \geq \Delta_{t}$ and $\eta^{-1} \Delta_{t} \geq \Delta_{t}$, then $\left\{l, \eta^{-1}, \eta^{-2}, \eta^{-3}, \ldots\right\}$, $\left\{l, \eta^{1}, \eta^{2}, \eta^{3}, \ldots\right\} \in X$, and hence by $\left(P_{4}\right)$,

$$
\left\{(\alpha, \beta) \in\left\{l, \eta, \eta^{2}, \eta^{3}, \ldots\right\} \times\left\{l, \eta^{-1}, \eta^{-2}, \eta^{-3}, \ldots\right\} \mid \alpha \beta=l\right\}
$$


must be finite. But then $\left\{l, \eta, \eta^{2}, \eta^{3}, \ldots\right\}$ must be finite, that is $\eta \in \Delta_{t}$ and thus $\eta \Delta_{t}=\Delta_{t}$. If $\alpha, \nu \in \Delta$, then by $\left(P_{1}\right),\left(P_{2}\right)$, and $\left(P_{5}\right)$, either $\left\{l,\left(\alpha \nu^{-1}\right)^{-1},\left(\alpha \nu^{-1}\right)^{-2},\left(\alpha \nu^{-1}\right)^{-3}, \ldots\right\} \in X$ or $\left\{l, \alpha \nu^{-1},\left(\alpha \nu^{-1}\right)^{2}\right.$, $\left.\left(\alpha \nu^{-1}\right)^{3}, \ldots\right\} \in X$. In the former case, $\alpha \Delta_{t} \geq \nu \Delta_{t}$; and in the latter case, $\nu \Delta_{t} \geq \alpha \Delta_{t}$. We conclude (cf. [8, page 13]) that $\left(\Delta / \Delta_{t}, \cdot, \geq\right)$ is a totally ordered group.

To see that $\succcurlyeq$ extends $\geq$, suppose that $\alpha \Delta_{t}>\nu \Delta_{t}$. Then $\alpha \nu^{-1} \tau>l$ for some $\tau \in \Delta_{t}$, and hence $\imath<\alpha \nu^{-1} \tau<\left(\alpha \nu^{-1} \tau\right)^{2}<\left(\alpha \nu^{-1} \tau\right)^{3}<\cdots$. But then, since $\tau \in \Delta_{t}$, there exists $n \in \mathbb{N}$ such that $l<\left(\alpha \nu^{-1}\right)^{n}<\left(\alpha \nu^{-1}\right)^{2 n}<$ $\left(\alpha \nu^{-1}\right)^{3 n}<\ldots$, and thus, since the elements of $X$ are locally inversely well-ordered, $\left\{l,\left(\alpha \nu^{-1}\right)^{n},\left(\alpha \nu^{-1}\right)^{2 n},\left(\alpha \nu^{1}\right)^{3 n}, \ldots\right\} \notin X$. By $\left(P_{2}\right)$, this implies that $\left\{l, \alpha \nu^{-1},\left(\alpha \nu^{-1}\right)^{2},\left(\alpha \nu^{-1}\right)^{3}, \ldots\right\} \notin X$ and hence, by $\left(P_{5}\right)$, that $\left\{l,\left(\alpha \nu^{-1}\right)^{-1},\left(\alpha \nu^{-1}\right)^{-2},\left(\alpha \nu^{-1}\right)^{-3}, \ldots\right\} \in X$. It follows that $\alpha \Delta_{t} \succ \nu \Delta_{t}$ and thus that $\succcurlyeq$ extends $\geq$.

We show last that $X / \Delta_{t} \subseteq I W(\succcurlyeq)$. Suppose by way of contradiction that $\Psi \subseteq A / \Delta_{t}$ for some $A \in X$ but that $\psi \notin I W(\succcurlyeq)$. Then there exists an infinite strictly increasing sequence $\cdots \succ \psi_{2} \Delta_{t} \succ \psi_{1} \Delta_{t}$ in $\Psi$. Since $\Psi \subseteq$ $A / \delta_{t}$, for every element $\Psi \Delta_{t} \in \Psi$, there exists $\alpha \in A$ such that $\alpha \Delta_{t}=\psi \Delta_{t}$, and hence by $\left(P_{2}\right)$ we may assume that $P=\left\{\psi_{1}, \psi_{2}, \ldots\right\} \in \operatorname{Cou}(X)$. By $\left(P_{5}\right)$ there exists $\mu \in P$ such that $\bigcup_{n=1}^{\infty}\left(\mu^{-1} P\right)^{n} \in X$. If $\psi_{k} \in P$ and $\psi_{k} \mu^{-1} \notin \Delta_{t}$, then

$$
\left.\left\{(\alpha, \beta) \in \bigcup_{n=1}^{\infty}\left(\mu^{-1} P\right)^{n}\right) \times\left\{l, \mu \psi_{k}^{-1},\left(\mu \psi_{k}^{-1}\right)^{2},\left(\mu \psi_{k}^{-1}\right)^{3}, \ldots\right\} \mid \alpha \beta=\imath\right\}
$$

is infinite, and hence by $\left(P_{4}\right),\left\{l, \mu \psi_{k}^{-1},\left(\mu \psi_{k}^{-1}\right)^{2},\left(\mu \psi_{k}^{-1}\right)^{3}, \ldots\right\} \notin X$. Then by $\left.\left(P_{5}\right),\left\{l, \mu \psi_{k}^{-1}\right)^{-1},\left(\mu \psi_{k}^{-1}\right)^{-2},\left(\mu \psi_{k}^{-1}\right)^{-3}, \ldots\right\} \in X$. If $\psi_{k} \in P$ and $\psi_{k} \mu^{-1} \in \Delta_{t}$, then $\left\{l,\left(\mu \sigma^{-1}\right)^{-1},\left(\mu \sigma^{-1}\right)^{-2},\left(\mu \sigma^{-1}\right)^{-3}, \ldots\right\}$ is finite and hence is in $X$ by $\left(P_{1}\right)$ and $\left(P_{2}\right)$. In both cases, we must have $\mu \Delta_{t} \succcurlyeq \psi_{k} \Delta_{t}$ for all $\psi_{k} \in P$. But then the sequence $\psi_{1} \Delta_{t}, \psi_{2} \Delta_{t}, \ldots$ cannot be strictly increasing. This is a contradiction and therefore we conclude that $X / \Delta_{t} \subseteq$ $I W(\succcurlyeq)$.

COROLlARY 1.8. If $(\Delta, \cdot, \geq)$ is a rooted abelian group and $X$ is a strongly supporting subset of $2^{\Delta}$, then $X / \Delta_{t}$ is a strongly supporting subset of $2^{\left(\Delta / \Delta_{\tau}, \cdot, \geq\right)}$.

Proof. By Proposition 1.7, $\succcurlyeq$ extends $\geq$ and $\left(\Delta / \Delta_{t}, \cdot, \succcurlyeq\right)$ is a totally ordered group. As we observed in the proof of Proposition 1.4, every element element of $I W(\geq)$ is a locally inversely well-ordered subset of $\left(\Delta / \Delta_{t}, \geq\right)$. Therefore, since $X / \Delta_{t} \subseteq I W I(\succcurlyeq)$ by Proposition $1.7, X / \Delta_{t}$ must satisfy 
$\left(P_{0}\right)$. Clearly $X / \Delta_{t}$ satisfies $\left(P_{1}\right)$ and $\left(P_{3}\right)$. To see that $X / \Delta_{t}$ satisfies $\left(P_{2}\right)$, note that, if $\Psi \subseteq A / \Delta_{t}$ for some $A \in X$, then $S=\left\{\alpha \in A \mid \alpha \Delta_{t} \in\right.$ $\Psi\} \in X$ and $\Psi=S / \Delta_{t}$ so that $\Psi \in X / \Delta_{t}$, and that, if $A, B \in X$, then $A / \Delta_{t} \cup B / \Delta_{t}=(A \cup B) / \Delta_{t} \in X / \Delta_{t}$. It is well-known (cf. [4] and [13]) that any pair of inversely well-ordered subsets of a totally ordered group satisfy $\left(P_{4}\right)$. Since $\left(\Delta / \Delta_{t}, \cdot, \geq\right)$ is a totally ordered group and $X / \Delta_{t} \subseteq I W(\succcurlyeq)$ by Proposition 1.7, it follows that $X / \Delta_{t}$ satisfies $\left(P_{4}\right)$. Since, for any $\alpha \in A \in$ $\left.X,\left[\bigcup n=1^{\infty}\left(\alpha^{-1} A\right)^{n}\right] / \Delta_{t}=\bigcup_{n=1}^{\infty}\left(\alpha \Delta_{t}\right)^{-1}\left(A / \Delta_{t}\right)\right]^{n}$, it follows $X / \Delta_{t}$ satisfies $\left(P_{5}\right)$.

Finally we note that, as a consequence of the results above, every strongly supporting collection is contained in a collection of the form described in Proposition 1.4.

Proposition 1.9. If $(\Delta, \cdot, \geq)$ is a rooted abelian group and $X$ is a strongly supporting subset of $2^{\Delta}$, then there exists an extended total order $\succcurlyeq$ on $\left(\Delta / \Delta_{t}, \cdot, \geq\right)$ such that $X \subseteq X(\succcurlyeq)$.

Proof. This result follows immediately from Proposition 1.7 and Corollary 1.6.

According to Proposition 1.4, every rooted abelian group has a supporting collection of the form $X(\succcurlyeq)$, and according to Proposition 1.9, every strongly supporting collection is contained in a strongly supporting collection of this form. This raises the question whether there are any strongly supporting collections not of the form $X(\succcurlyeq)$. The collection of finite subsets is certainly supporting. However, if the group is torsion, then this collection is clearly of the form $X(\succcurlyeq)$, and if the group has even one element of infinite order, then this collection is not strongly supporting (cf. the proof of Proposition 4.4 below). In spite of this, however, there do exist strongly supporting collections which are not of the form $X(\succcurlyeq)$. One of these is described in the next example.

EXAMPLE 1.10. Let $(\Delta, \cdot, \geq)$ be a totally ordered group which contains an uncountable inversely well-ordered subset and consider $X=\operatorname{Cou}(I W(\geq))$. Since $\Delta$ is totally ordered, the only extended total order on $\Delta / \Delta_{t}=\Delta$ is $\geq$ itself and $X(\geq)=I W(\geq)$. Therefore, since $X \neq I W(\geq)$ be our choice of $\Delta, X \neq X(\succcurlyeq)$ for any extended total order $\succcurlyeq$ on $\Delta / \Delta_{t}$. But $X$ clearly satisfies $\left(P_{0}\right),\left(P_{1}\right)$ and $\left(P_{2}\right)$; since the product of countable sets is certainly countable, $X$ satisfies $\left(P_{3}\right)$; since $X$ is contained in the set $I W(\geq)$ which satisfies $\left(P_{4}\right)$ by Proposition $1.1, X$ satisfies $\left(P_{4}\right)$; and finally, since the set $I W(\geq)$ both contains $X$ and satisfies $\left(P_{5}\right)$ and since the countable union 
of countable sets is countable, $X$ satisfies $\left(P_{5}\right)$. Thus, $X$ is a strongly supporting collection which is not of the form $X(\succcurlyeq)$ for any extended total order $\succcurlyeq$ on $\Delta / \Delta_{t}$.

\section{Power series algebras}

We first recall the general construction of power series algebras over a totally ordered field.

Let $(\Delta, \cdot, \geq)$ be a rooted abelian group with identity $l$, let $X$ be a supporting subset of $2^{\Delta}$, and let $G$ be a totally ordered field. Form the product $\prod_{\Delta} T$; for $\alpha \in \Delta$ and $r \in L$, let $\bar{\alpha}, \bar{r} \in \prod_{\Delta} t$ be the vectors

$$
\bar{\alpha}_{\delta}=\left\{\begin{array}{ll}
1 & \text { if } \delta=\alpha \\
0 & \text { otherwise },
\end{array} \bar{r}_{\delta}= \begin{cases}r & \text { if } \delta=\imath \\
0 & \text { otherwise }\end{cases}\right.
$$

let $\bar{\Delta}=\{\bar{\alpha} \mid \alpha \in \Delta\}$. For $v \in \prod_{\Delta} T$, let $\operatorname{Supp}(v)=\left\{\delta \in \Delta \mid v_{\delta} \neq 0\right\}$ denote the support of $v$ and let ${ }_{X} \prod_{\Delta} T$ denote the set of all vectors $v \in \Pi_{\Delta} T$ such that $\operatorname{Supp}(v) \in X$. Define addition on ${ }_{X} \Pi_{\Delta} T$ coordinatewise:

$$
(u+v)_{\delta}=u_{\delta}+v_{\delta}
$$

define multiplication on ${ }_{x} \prod_{\Delta} T$ via convolution:

$$
(u v)_{\delta}=\sum_{\alpha \beta=\delta} r_{\alpha, \beta} u_{\alpha} v_{\beta},
$$

where $\left\{r_{\alpha, \beta}\right\} \subseteq T$ is a factor set:

(a) $r_{\alpha, \beta}>0$ for all $\alpha, \beta \in \Delta$;

(b) $r_{\alpha \beta, \gamma} r_{\alpha, \beta}=r_{\alpha, \beta \gamma} r_{\beta, \gamma}$ for all $\alpha, \beta, \gamma \in \Delta$;

(c) $r_{\delta, t}=1=r_{t, \delta}$ for all $\delta \in \Delta$;

(d) $r_{\alpha, \beta}=r_{\beta, \alpha}$ for all $\alpha, \beta \in \delta$.

The resulting structure, $\left({ }_{X} \prod_{\Delta} T,\left\{r_{\alpha, \beta}\right\},+, \cdot\right)$, is a commutative ring with $\bar{\imath}=\overline{1}$ as the multiplicative identity (cf. [4], [5], [13], [16], [20], [21], [22]). With the pointwise scalar multiplication: $(t u)_{\delta}=t u_{\delta}$ for all $t \in T, u \in$ ${ }_{x} \prod_{\Delta} T$ and $\delta \in \Delta,\left({ }_{X} \prod_{\Delta} T,\left\{t_{\alpha, \beta}\right\},+, \cdot\right)$ is clearly a commutative algebra over $T$.

If $u \in{ }_{X} \Pi_{\Delta} T$ and $\sigma \in \operatorname{Supp}(u)$, then, since $\operatorname{Supp}(u)$ is locally inversely well-ordered, there is a maximal element $\mu \in \operatorname{Supp}(u)$ such that $\mu \geq \sigma$. We may thus define a binary relation $>$ on ${ }_{X} \prod_{\Delta} T$ as follows:

$u>0$ if and only if $u_{\mu}>0$ for all maximal elements $\mu \in \operatorname{Supp}(u)$;

$$
u>v \text { if and only if } u-v>0 \text {. }
$$


The resulting structure, $\left({ }_{X} \prod_{\Delta} T,\left\{r_{\alpha, \beta}\right\},+\cdot, \geq\right)$, is a commutative latticeordered algebra over $T$, where for all $u \in{ }_{X} \prod_{\Delta} T$,

$$
(u \vee 0)_{\delta}= \begin{cases}u_{\delta} & \text { if } u_{\mu}>0, \text { where } \mu \geq \delta \text { is maximal in } \operatorname{Supp}(u) \\ 0 & \text { otherwise. }\end{cases}
$$

(See [4], [5, Page 0.12], [9], [13], [16], [17], [18], [19], [20], [21], [22]. When Ribenboim's convolution rings [20], [21], [22] are based on a group, they are a special case of this construction; he requires the supports to be locally inversely well-ordered and to have no infinite disjoint subsets.) If $(\Delta, \geq)$ is trivially ordered, that is, if $\alpha=\beta$ whenever $\alpha \geq \beta, \geq$ is the pointwise order on ${ }_{X} \prod_{\Delta} L$, and hence for all $u \in{ }_{X} \prod_{\Delta} L,(u \vee 0)_{\delta}=u_{\delta} \vee 0$ (cf. [2], [5], [8]).

The construction given above is very general and, because of the necessity of choosing a supporting collection, somewhat arbitrary. To describe less arbitrary cases of this construction, suppose that $(\Delta, \cdot, \geq)$ is a rooted abelian group and that $T$ is a totally ordered field.

(A) If $F$ denotes the collection of finite subsets of $\Delta$, then clearly $F$ is supporting. Since the elements in the resulting power series algebra are precisely those vectors with finite support, a power series algebra determined by $F$ is denoted by $\left(\Sigma_{\Delta} T,\left\{r_{\alpha, \beta}\right\},+, \cdot\right)$.

(B) If $\geq$ is a total order on $\Delta$, then by Proposition 1.1 IW( $\geq)$ is a strongly supporting subset, and hence, for any factor set $\left\{t_{\alpha, \beta}\right\} \subseteq T$, we may use it to form a lattice-ordered power series algebra, usually denote by $\left(\geq \prod_{\Delta} T,\left\{t_{\alpha, \beta}\right\},+, \cdot, \geq\right.$ ). It is well-known (cf. [13] and [8, page 137, Theorem 10]) that the resulting structure is a totally ordered field.

(C) In [18], it was shown how to construct a lattice-ordered power series field over a torsion-free rooted abelian group. This process may be generalized to an arbitrary rooted abelian group $(\Delta, \cdot, \geq)$ as follows. By Proposition $1.2,\left(\Delta / \Delta_{t}, \cdot, \geq\right)$ is a torsion-free rooted abelian group and hence has an extended total order $\succcurlyeq$. By Proposition 1.4, this total order generators a strongly supporting collection $X(\succcurlyeq) \subseteq 2^{\Delta}$. For any factor set $\left\{r_{\alpha, \beta}\right\} \subseteq T$, we may thus form a lattice-ordered power series algebra, usually denoted by $\left(\succcurlyeq \prod_{\Delta} T,\left\{t_{\alpha, \beta}\right\}, \cdot, \geq\right)$. Note that there is no ambiguity in notation between (B) and (C). For if $(\Delta, \cdot, \geq)$ is totally ordered, then $\succcurlyeq$ must coincide with $\geq$ (cf. Example 1.10) and $\succcurlyeq \Pi_{\Delta} T$ must coincide with $\geq \Pi_{\Delta} T$.

We first observe that any lattice-ordered power series algebra generated by a strongly supporting collection is contained in an algebra of the form described in (C).

Proposition 2.1. If $T$ is a totally ordered field, if $(\Delta, \cdot, \geq)$ is a rooted abelian group, and if $X$ is a strongly supporting subset of $2^{\Delta}$, then there exists an extended total order $\succcurlyeq$ on $\left(\Delta / \Delta_{t}, \cdot, \geq\right)$ such that for any factor 
set $\left\{t_{\alpha, \beta}\right\} \subseteq T,\left({ }_{X} \Pi_{\Delta}, T,\left\{t_{\alpha, \beta}\right\},+, \cdot, \geq\right)$ is a convex lattice subalgebra of $\left(_{\succcurlyeq} \prod_{\Delta} T,\left\{t_{\alpha, \beta}\right\},+, \cdot, \geq\right)$.

Proof. This is an immediate consequence of Proposition 1.9 and the properties of strongly supporting subsets.

If $\Delta$ is a torsion-free, then a lattice-ordered power series algebra of the form $\left({ }_{\preccurlyeq} \prod_{\Delta} T,\left\{t_{\alpha, \beta}\right\},+, \cdot, \geq\right)$ is just the usual power series field as described in (B) with a lattice order rather than the usual total order. This process was described in detail in [18], where it was also shown how to vary the construction to create examples of lattice-ordered fields in which $1 \ngtr 0$. The other extreme case occurs when $\Delta$ is torsion. In that case, the power series algebra must be of the form $\sum_{\Delta} T$.

Proposition 2.2. If $T$ is a totally ordered field, if $(\Delta, \cdot, \geq)$ is a torsion rooted abelian group, and if $X$ is a strongly supporting subset of $2^{\Delta}$, then for any factor set $\left\{t_{\alpha, \beta}\right\} \subseteq T,\left({ }_{X} \prod_{\Delta} T,\left\{t_{\alpha, \beta}\right\},+, \cdot, \geq\right)=\left(\sum_{\Delta} T,\left\{t_{\alpha, \beta}\right\},+, \cdot\right.$, $\geq$ ).

Proof. By $\left(P_{1}\right), \sum_{\Delta} T \subseteq{ }_{X} \prod_{\Delta} T$, and by Proposition 2.1, there exists an extended total order $\succcurlyeq$ on $\Delta / \Delta_{t}$ such that ${ }_{X} \prod_{\Delta} T \subseteq \succcurlyeq \Pi_{\Delta} T$. It therefore suffices to show that $\succcurlyeq \prod_{\Delta} T \subseteq \sum_{\Delta} T$. But, by definition, since $\Delta$ is torsion $X(\succcurlyeq)$ is precisely the set of finite subsets of $\Delta$, and hence in fact, $\succcurlyeq \Pi_{\Delta} T=$ $\sum_{\Delta} T$.

\section{Power series fields}

To this point, we have considered the structures ${ }_{x} \prod_{\Delta} T$ as algebras; we now want to consider them as fields. Our object in this section is to determine when power series algebras of the form $\left(_{\succcurlyeq} \prod_{\Delta} T,\left\{t_{\alpha, \beta}\right\},+, \cdot\right)$ are fields and thus when lattice-ordered power series algebras of the form $\left(_{\succcurlyeq} \prod_{\Delta} T,\left\{t_{\alpha, \beta}\right\},+, \cdot, \geq\right)$ are lattice-ordered fields. We begin by considering the elements with torsion support.

We first note that such elements are algebraic over $T$. For the proof of this result, we use the notation $o(\alpha)$ to denote the order of the element $\alpha$ of the group $\Delta$.

Proposition 3.1. Suppose that $T$ is a totally ordered field, that $\left\{t_{\alpha, \beta}\right\} \subseteq T$ is a factor set, that $(\Delta, \cdot, \geq)$ is a rooted abelian group, and that $\succcurlyeq$ is an extended total order on $\left(\Delta / \Delta_{t}, \cdot, \geq\right)$. If $\left.\succcurlyeq \prod_{\Delta} T\right|_{t}=\left\{v \in_{\succcurlyeq} \Pi_{\Delta} T \mid \operatorname{Supp}(v) \subseteq\right.$ 
$\left.\Delta_{t}\right\}$, then $\succcurlyeq \prod_{\Delta} T / t$ is a convex lattice subalgebra of $\succcurlyeq \Pi_{\Delta} T$ which is algebraic over $T$.

Proof. Clearly $\left.\succcurlyeq \Pi_{\Delta} T\right|_{t}$ is a convex lattice subalgebra of $\geq \Pi_{\Delta}$. Observe that for any $\alpha \in \Delta$ and any $n>0, \bar{\alpha}^{n}=t_{\alpha, \alpha} \cdots t_{\alpha, \alpha^{n-1}} \overline{\alpha^{n}}$. Thus, if $\tau \in \Delta_{t}$, then $\bar{\tau}$ is a root of the polynomial $x^{o(\tau)}-t_{\tau, \tau} \cdots t_{\tau, \tau}^{o(t)-1} \in T[x]$ and hence $\bar{\tau}$ is algebraic over $T$. If $\left.v \in_{\succcurlyeq} \prod_{\Delta} T\right|_{t}$, then $\operatorname{Supp}(v)$ is finite by definition of $X(\succcurlyeq)$, and hence $v$ is a finite linear combination of elements of the form $\bar{\tau}$. It follows that $v$ is algebraic over $T$.

We note next that when an index group is torsion, we are really dealing with only one power series field.

Proposition 3.2. Suppose that $T$ is a totally ordered field, that $\left\{t_{\alpha, \beta}\right\} \subseteq T$ is a factor set, that $(\Delta, \cdot, \geq)$ is a rooted abelian group, and that $\succcurlyeq$ is an extended total order on $\left(\Delta / \Delta_{t}, \cdot, \geq\right)$. Then $\left.\succcurlyeq \prod_{\Delta} T\right|_{t}=\sum_{\Delta_{t}} T$. If $\Delta$ is torsion, then $\succcurlyeq \Pi_{\Delta} T=\sum_{\Delta} T$.

Proof. This result follows from Corollary 1.6 and Proposition 2.2.

By Proposition 3.2, the following two results characterize both those situations in which $\succcurlyeq \prod_{\Delta} T$ is a field for a torsion index group $\Delta$ and those in which $\left.\succcurlyeq \prod_{\Delta} T\right|_{t}$ is a field for an arbitrary index group $\Delta$. The first result (Proposition 3.3) determines when a given power series algebra over a torsion index group is a field; the second (Proposition 3.4) determines when, for a given torsion group and totally ordered field, there exists a factor set allowing the construction of a power series field.

For the results which follow, we need the notation $F^{a}$ for the algebraic closure of a field $F$.

Proposition 3.3. For a totally ordered field $T$, a factor set $\left\{t_{\alpha, \beta}\right\} \subseteq T$, and $a$ torsion abelian group $\Delta$, the following statements are equivalent:

(i) $\left(\sum_{\Delta} T,\left\{t_{\alpha, \beta}\right\},+, \cdot\right)$ is a field;

(ii) $\left(\sum_{\Delta} T,\left\{t_{\alpha, \beta}\right\},+, \cdot\right)$ has no zero-divisors;

(iii) there exists a one-to-one function $i: \Delta \rightarrow T^{a *}$ such that

(1) $\{i(\tau) \mid \tau \in \Delta\}$ is a linearly independent subset of $T^{a}$ over $T$;

(2) for all $\sigma, \tau \in \Delta, t_{\sigma, \tau}=i(\sigma) i(\tau) i(\sigma \tau)^{-1}$.

Proof. By Propositions 3.1 and 3.2, $\sum_{\Delta} T$ is an algebraic over $T$. It is well-known (cf. [10, page 8 , \#VII]) that in this case, (i) is equivalent to (ii). 
(i) $\Rightarrow$ (iii). Since $\Delta$ is a rooted abelian group with respect to the trivial order, Proposition 3.1 allows us to define a map $i: \Delta \rightarrow T^{a *}$ by letting $i(\tau)=\bar{\tau}$. Clearly $\{\bar{\tau} \mid \tau \in \Delta\}$ is a linearly independent subset of $\sum_{\Delta} T$ over $T$ and hence of $T^{a}$ over $T$. As well, $i(l)=\bar{\imath}=1$, and for $\sigma, \tau \in \Delta$,

$$
i(\sigma) i(\tau) i(\sigma \tau)^{-1}=\overline{\sigma \tau \sigma \tau}^{-1}=t_{\sigma, \tau} \overline{\sigma \tau} \overline{\sigma \tau}^{-1}=t_{\sigma, \tau} .
$$

(iii) $\Rightarrow$ (ii) Since $\left\{t_{\alpha, \gamma}\right\}$ is a factor set, we may form the lattice-ordered power series algebra $\left(\sum_{\Delta} T,\left\{t_{\alpha, \beta}\right\},+, \cdot, \geq\right)$. Define $I: \sum_{\Delta} T \rightarrow T^{a}$ by letting $I\left(a_{1} \bar{\alpha}_{1}+\cdots+a_{n} \bar{\alpha}_{n}\right)=a_{1} i\left(\alpha_{1}\right)+\cdots+a_{n} i\left(\alpha_{n}\right)$. Clearly $I$ preserves addition and scalar multiplication, and since

$I(\bar{\alpha} \bar{\gamma})=I\left(t_{\alpha, \gamma} \overline{\alpha \gamma}\right)=t_{\alpha, \beta} i(\alpha \gamma)=i(\alpha) i(\gamma) i(\alpha \gamma)^{-1} i(\alpha \gamma)=i(\alpha) i(\gamma)=I(\bar{\alpha}) I(\bar{\gamma})$, $I$ also preserves multiplication. By (1), $I$ establishes a one-to-one ringhomomorphism from $\sum_{\Delta} T$ into $T^{a}$. But, since $T^{a}$ is a field, it has no divisors of zero, and therefore $\sum_{\Delta} T$ has no divisors of zero.

Proposition 3.4. For a totally ordered field $T$ and a torsion abelian group $\Delta$, the following statements are equivalent:

(i) there exists a factor set $\left\{t_{\alpha, \beta}\right\} \subseteq T$ such that $\left(\sum_{\Delta} T,\left\{t_{\alpha, \beta}\right\},+\cdot\right)$ is a field;

(ii) there exists a one-to-one function $i: \Delta \rightarrow T^{a *}$ such that

(1) $\{i(\tau) \mid \tau \in \Delta\}$ is a linearly independent subset of $T^{a}$ over $T$;

(2) $\pi \circ i: \Delta \rightarrow T^{a *} / T^{+}$is a group-homomorphism, where $\pi: T^{a *} \rightarrow$ $T^{a *} / T^{+}$is the canonical epimorphism.

Proof. (i) $\Rightarrow$ (ii). Let $i: \Delta \rightarrow T^{a *}$ be a function satisfying the conditions of Proposition 3.3 (iii). Those conditions state that $i$ is one-to-one and (1) holds. To see that (2) holds, observe that

$$
i(\sigma) i(\tau) T^{+}=i(\sigma) i(\tau) i(\sigma \tau)^{-1} i(\sigma \tau) T^{+}=t_{\sigma, \tau} i(\sigma \tau) T^{+}=i(\sigma \tau) T^{+} .
$$

(ii) $\Rightarrow$ (i). For $\sigma, \tau \in \Delta$, let $t_{\sigma, \tau}=i(\sigma) i(\tau) i(\sigma \tau)^{-1} i(l)^{-1}$. By (2), $\left\{t_{\alpha, \beta}\right\} \subseteq$ $T$; we claim that $\left\{t_{\alpha, \gamma}\right\}$ is a factor set. By (2), $t_{\alpha, \gamma}>0$ and $t_{\alpha, l}=1=t_{t, \gamma}$, and since $T^{a}$ is commutative, $t_{\alpha, \gamma}=t_{\gamma, \alpha}$. Finally,

$$
\begin{aligned}
t_{\alpha \gamma, \sigma} t_{\alpha, \gamma} & =i(\alpha \gamma) i(\sigma) i(\alpha \gamma \sigma)^{-1} i(l)^{-1} i(\alpha) i(\gamma) i(\alpha \gamma)^{-1} i(l)^{-1} \\
& =i(\alpha) i(\alpha \gamma \sigma)^{-1} i(l)^{-1} i(\gamma) i(\sigma) i(l)^{-1} \\
& =i(\alpha) i(\gamma \sigma) i(\alpha \gamma \sigma)^{-1} i(l)^{-1} i(\gamma) i(\sigma) i(\gamma \sigma)^{-1} i(l)^{-1}=t_{\alpha, \gamma \sigma} t_{\gamma, \sigma} .
\end{aligned}
$$

Therefore $\left\{t_{\alpha, \gamma}\right\}$ is a factor set and that (ii) implies (i) follows immediately from Proposition 3.3.

Our next step is to show that the above results characterize the fields $\succcurlyeq \Pi_{\Delta} T$ is the sense that $\succcurlyeq \Pi_{\Delta} T$ is a field whenever $\left.\succcurlyeq \Pi_{\Delta} T\right|_{t}$ is a field. 
Proposition 3.5. Suppose that $T$ is a totally ordered field, that $\left\{t_{\alpha, \beta}\right\} \subseteq T$ is a factor set, that $(\Delta, \cdot, \geq)$ is a rooted abelian group, and that $\succcurlyeq$ is an extended total order on $\left(\Delta / \Delta_{t}, \cdot, \geq\right)$. Then $\succcurlyeq \prod_{\Delta} T$ is a field ifand only if $\left.\succcurlyeq \Pi_{\Delta} T\right|_{t}$ is a field.

Proof. By Proposition 3.1, $\left.\Pi_{\Delta} T\right|_{t}$ is an algebraic subalgebra of $\succcurlyeq \Pi_{\Delta} T$, and hence if $\succcurlyeq \Pi_{\Delta} T$ is a field, then $\succcurlyeq \Pi_{\Delta} T / t$ must also be a field.

Conversely, suppose that $\succcurlyeq \prod_{\Delta} T / t$ is a field and let $0 \neq v \in \succcurlyeq \Pi_{\Delta} T$. Then there exist $\mu \in \Delta, a \in \succcurlyeq \prod_{\Delta} T / t$, and $v^{-} \in \succcurlyeq \prod_{\Delta} T$ such that $v=a \bar{\mu}\left(1+v^{\wedge}\right)$ and for all $\delta \in \operatorname{Supp}\left(v^{\wedge}\right), \Delta_{t} \succ \delta \Delta_{t}$. Observe firstly that by Lemma 0.1, $\left[\bigcup_{n=1}^{\infty} \operatorname{Supp}\left(v^{\wedge}\right)^{n}\right] / \Delta_{t}=\bigcup_{n=1}^{\infty}\left(\operatorname{Supp}\left(v^{\wedge}\right) / \Delta_{t}\right)^{n} \in I W(\succcurlyeq)$ and for all $\delta \in \Delta$, there exist only finitely many $n$ such that $\delta \Delta_{t} \in \operatorname{Supp}\left(v^{\wedge}\right)^{n} / \Delta_{t}$. Observe secondly that by $\left(P_{3}\right), \operatorname{Supp}\left(v^{\wedge}\right)^{n} \in X(\succcurlyeq)$ for all $n$ and hence that $\operatorname{Supp}\left(v^{-}\right)^{n} \cap \delta \Delta$ is finite for all $n$ and all $\delta \in \Delta$. Then by Lemma 1.3, $\left[\bigcup_{n=1}^{\infty} \operatorname{Supp}\left(v^{-}\right)^{n}\right] \cap \delta \Delta_{t}$ is finite for all $\delta \in \Delta$, and we conclude that $\bigcup_{n=1}^{\infty} \operatorname{Supp}\left(v^{\wedge}\right)^{n} \in X(\succcurlyeq)$. As well, if $\gamma \in \operatorname{Supp}\left(v^{\wedge}\right)^{n}$ for some $n$, then $\gamma \Delta_{t} \in \operatorname{Supp}\left(v^{\wedge}\right)^{n} / \Delta_{t}$, and hence for all $\gamma \in \Delta$, there exist only finitely many $n$ such that $\gamma \in \operatorname{Supp}\left(v^{\wedge}\right)^{n}$. We conclude that $\sum_{n=1}^{\infty}\left(-v^{-}\right)^{n}$ is well-defined and is an element of $\succcurlyeq \Pi_{\Delta} T$ and hence that $\left(1+v^{\wedge}\right)^{-1}$ exists in $\succcurlyeq \Pi_{\Delta} T$. By hypothesis, $a^{-1}$ exists in $\succcurlyeq \Pi_{\Delta} T / t$ and hence in $\succcurlyeq \Pi_{\Delta} T$. Clearly $\left(t_{\mu, \mu^{-1}}\right)^{-1} \overline{\mu^{-1}} \in \succcurlyeq \Pi T$ and hence $\bar{\mu}^{-1}$ exists in $\succcurlyeq \Pi_{\Delta} T$. It follows that $v^{-1}=a^{-1} \bar{\mu}^{-1}\left(1+v^{-}\right)^{-1} \in{ }_{\succ} \Pi_{\Delta} T$.

We are now in a position to characterize those power series algebras of the form $\left(\succcurlyeq \prod_{\Delta} T,\left\{t_{\alpha, \beta}\right\},+, \cdot\right)$ which are fields and thus those lattice-ordered power series algebras of the form $\left(\succcurlyeq \Pi_{\Delta} T,\left\{t_{\alpha, \beta}\right\},+, \cdot, \geq\right)$ which are latticeordered fields. As above, our characterization is phrased in two ways: the first (Corollary 3.6) describes when a given power series algebra is a field; the second (Corollary 3.7) describes when, for a given group and totally ordered field, there exists a factor set allowing the construction of a power series field.

COROLLARY 3.6. For a totally ordered field $T$, a factor set $\left\{t_{\alpha, \beta}\right\} \subseteq T$, an extended total order $\succcurlyeq$ on $\Delta / \Delta_{t}$, and a rooted abelian group $(\Delta, \cdot, \geq)$, the following statements are equivalent:

(i) $\left(\succcurlyeq \prod_{\Delta} T,\left\{t_{\alpha, \beta}\right\},+, \cdot\right)$ is a field;

(ii) $\left(\left.\succcurlyeq \prod_{\Delta} T\right|_{t},\left\{t_{\alpha, \beta}\right\},+, \cdot\right)$ has no zero-divisors;

(iii) there exists a one-to-one function $i: \Delta_{t} \rightarrow T^{a *}$ such that

(1) $\left\{i(\tau) \mid \tau \in \Delta_{t}\right\}$ is a linearly independent subset of $T^{a}$ over $T$;

(2) for all $\sigma, \tau \in \Delta_{t}, t_{\sigma, \tau}=i(\sigma) i(\tau) i(\sigma \tau)^{-1}$. 
Proof. The equivalence of these statements follows immediately from Propositions 3.2, 3.3, and 3.5.

COROLlaRY 3.7. Suppose that $T$ is a totally ordered field, that $(\Delta, \cdot, \geq)$ is a rooted abelian group, and that $\succcurlyeq$ is an extended total order on $\left(\Delta / \Delta_{t}, \cdot, \geq\right)$. Then the following statements are equivalent:

(i) there exists a factor set $\left\{t_{\alpha, \beta}\right\} \subseteq T$ such that $\left(_{\succcurlyeq} \prod_{\Delta} T,\left\{t_{\alpha, \beta}\right\},+, \cdot\right)$ is a field;

(ii) there exists a one-to-one function $i: \Delta_{t} \rightarrow T^{a *}$ such that

(1) $\left\{i(\tau) \mid \tau \in \Delta_{t}\right\}$ is a linearly independent subset of $T^{a}$ over $T$;

(2) $\pi \circ i: \Delta_{t} \rightarrow T^{a *} / T^{+}$is a group-homomorphism, where $\pi: T^{a *} \rightarrow$ $T^{a *} / T^{+}$is the canonical epimorphism.

Proof. The equivalence of these statements follows immediately from Propositions 3.2, 3.4, and 3.5.

A final application of Proposition 3.5 is the following.

CoROLlary 3.8. Suppose that $T$ is a totally ordered field, that $\left\{t_{\alpha, \beta}\right\} \subseteq T$ is a factor set, that $(\Delta, \cdot, \geq)$ is a rooted abelian group, that $\succcurlyeq$ is an extended total order on $\left(\Delta / \Delta_{t}, \cdot, \geq\right)$, and that $X$ is a strongly supporting subset of $2^{\Delta}$. If ${ }_{X} \prod_{\Delta} T$ is a field, then so is $\succcurlyeq \prod_{\Delta} T$.

Proof. By Corollary 1.6 and $\left(P_{1}\right)$ and $\left(P_{2}\right),\left.\succcurlyeq \prod_{\Delta} T\right|_{t}$ is a subalgebra of ${ }_{X} \prod_{\Delta} T$; by Proposition 3.1, all the elements of $\left.\succcurlyeq \prod_{\Delta} T\right|_{t}$ are algebraic over $T$. Since ${ }_{x} \Pi_{\Delta} T$ is a field, it follows that $\left.\succcurlyeq \Pi_{\Delta} T\right|_{t}$ is a field and hence, by Proposition 3.5, that $\succcurlyeq \prod_{\Delta} T$ is a field.

\section{Embeddings of lattice-ordered fields}

Any lattice-ordered field $L$ in which $1>0$ has a unique maximal totally ordered subfield $M(L)$. There are several different proofs of this-see [14] (cf. [15]), [24], and [19]. In [19], the embeddability of $L$ in a power series algebra over $M(L)$ was characterized in over twenty different ways. One of those ways is given in the first result below.

To state this result, recall the following terminology and notation (cf. [2] and [5]). An element $b$ of a lattice-ordered group $G$ is basic if $0<b$ and $[0, b]$ is totally ordered; if every positive element of $G$ exceeds a basic element, then any maximal disjoint set of basic elements of $G$ is called a 
basis for $G$ and $G$ is said to have a basis; the set of basic elements of any lattice-ordered group $G$ is denoted by $B(G)$. (Note that since a basis for a lattice-ordered field in the sense defined above need not be a basis of the field as a vector space over some subfield, this terminology is ambiguous to a certain extent. In the sequel, we will avoid this ambiguity by using the word "basis" exclusively in the order-theoretic sense defined above.) For any $S \subseteq G, S^{\perp}=\{z \in G|| z|\wedge| s \mid=0$ for all $s \in S\}$; if $g \in G$, then $g^{\perp}=\{g\}^{\perp}$. If $G$ has a basis $B$ and if $g \in G$, then $B(g)=\{b \in B \mid g \wedge b \neq 0\}=B \cap g^{\perp \perp}$. An element $t$ of a lattice-ordered field $L$ is said to be totally positive if both $0<t$ and $0<t^{-1}$.

Proposition 4.1. For a lattice-ordered field $L$ in which $1>0$, the following statements are equivalent.

(i) There exist a rooted abelian group $(\Delta, \cdot, \geq)$, a supporting subset $X$ of $2^{\Delta}$, a factor set $\left\{m_{\alpha, \beta}\right\} \subseteq M(L)$, and a one-to-one homomorphism $\lambda: L \rightarrow\left({ }_{X} \prod_{\Delta} M(L),\left\{m_{\alpha, \beta}\right\},+, \cdot, \geq\right)$ of lattice-ordered algebras over $M(L)$ such that $\lambda(L)$ contains $\bar{\Delta}$.

(ii) The basic elements of $L$ are totally positive; there exists a basis $B$ of $L$ such that $b^{\perp \perp}$ is unbounded for all $b \in B$; and for all positive elements $u, v$ of $L, B(u v) \subseteq(B(u) B(v))^{\perp \perp}$ and $\{(a, c) \in B(u) \times$ $\left.B(v) \mid a c \in 1^{\perp \perp}\right\}$ is finite.

Proof. This result follows from [19, Proposition 7.2]

One obvious question raised by this result is when the algebra of condition (i) is a field. Using the results of Section 3, we have the following partial answer to this question.

Proposition 4.2. Let $L$ be a lattice-ordered field in which $1>0$, and suppose that $L$ satisfies the following conditions: the basic elements of $L$ are totally positive; there exists a basis $B$ of $L$ such that $b^{\perp \perp}$ is unbounded for all $b \in B$; for all positive elements $u$, $v$ of $L, B(u v) \subseteq(B(u) B(v))^{\perp \perp}$ and $\left\{(a, c) \in B(u) \times B(v) \mid(a c)^{\perp \perp}=1^{\perp \perp}\right\}$ is finite; and if $C$ is a countable subset of $B(u)$ for some $u \in L$, then there exist $c \in C$ and $v \in L$ such that $\bigcup_{n=1}^{\infty}\left(c^{-1} C\right)^{n} \subseteq B(v)$. Then there exist a rooted abelian group $(\Delta, \cdot, \geq)$, an extended total order $\succcurlyeq$ on $\left(\Delta / \Delta_{t}, \cdot, \geq\right)$, a factor set $\left\{m_{\alpha, \beta}\right\} \subseteq M(L)$, and a one-to-one homomorphism $\lambda: L \rightarrow\left(\succcurlyeq \prod_{\Delta} M(L),\left\{m_{\alpha, \beta}\right\},+, \cdot, \geq\right)$ of lattice-ordered fields such that $\lambda(L) \supseteq \sum_{\Delta} M(L)$.

Proof. By Proposition 4.1, there exist a rooted abelian group $(\Delta, \cdot, \geq)$, a 
supporting subset $X$ of $2^{\Delta}$, a factor set $\left\{m_{\alpha, \beta}\right\} \subseteq M(L)$, and a one-to-one homomorphism $\lambda: L \rightarrow\left({ }_{X} \prod_{\Delta} M(L),\left\{m_{\alpha, \beta}\right\},+, \cdot, \geq\right)$ of lattice-ordered algebras over $M(L)$ such that $\lambda(L)$ contains $\bar{\Delta}$. Note that by [19, Proposition 3.5], if $M(L)^{c}=\{z \in L \mid 0<s \leq z \leq t$ for some $s, t \in M(L)\}$, then $M(L)^{c}$ is a convex subgroup of $B(L)$, and that by [19, Theorem 7.1] and its proof, we may assume that $\Delta=B(L) / M(L)^{c}$ and that $X$ is the set of all $A \subseteq \Delta$ for which there exists $0<u \in L$ such that $A \subseteq\left\{b M(L)^{c} \mid b \in B(u)\right\}$. However, the final condition on $L$ clearly implies that $X$ is strongly supporting and hence by Proposition 2.1 that there exists an extended total order $\succcurlyeq$ on $\left(\Delta / \Delta_{t}, \cdot, \geq\right)$ such that $\left({ }_{X} \Pi_{\Delta} M(L),\left\{m_{\alpha, \beta}\right\},+, \cdot, \geq\right)$ is a convex lattice subalgebra of $\left(\succcurlyeq \prod_{\Delta} M(L),\left\{m_{\alpha, \beta}\right\},+, \cdot \geq\right)$. We use Corollary 3.6 to complete the proof. By Proposition 3.1, if $\tau \in \Delta_{t}$, then $\bar{\tau}$ is algebraic over $M(L)$. This we may define $i: \Delta_{t} \rightarrow M(L)^{a *}$ by letting $i(\tau)=\bar{\tau}$. Since $\lambda$ is one-to-one, $i$ is one-to-one and clearly $\left\{i(\tau) \mid \tau \in \Delta_{t}\right\}$ is linearly independent over $M(L)$. As well, for $\sigma, \tau \in \Delta_{t}$,

$$
i(\sigma) i(\tau) i(\sigma \tau)^{-1}=\overline{\sigma \tau \sigma \tau}{ }^{-1}=t_{\sigma, \tau} \bar{\sigma} \bar{\tau} \bar{\sigma} \bar{\tau}^{-1}=t_{\sigma, \tau} .
$$

It follows from Corollary 3.6 that $\left(\prod_{\Delta} M(L),\left\{m_{\alpha, \beta}\right\},+, \cdot, \geq\right)$ is a field.

The additional condition in Proposition 4.2 is unwieldy and inelegant, and for this reason it would be best if it could be eliminated. Proposition 4.4 and Corollary 4.5 below go a step in this direction by showing that the other conditions do indeed imply a similar, albeit weaker, version of the additional condition.

LEMmA 4.3. Let $T$ be a totally ordered field, let $\left\{t_{\alpha, \beta}\right\} \subseteq T$ be a factor set, let $(\Delta, \cdot, \geq)$ be a rooted abelian group, and let $X$ be a supporting subset of $2^{\Delta}$. If $\varepsilon \in \Delta$ and $u=(1+\bar{\varepsilon})^{-1}$ exists in $\left({ }_{X} \prod_{\Delta} T,\left\{t_{\alpha, \beta}\right\},+, \cdot, \geq\right)$, then either $\left\{\imath, \varepsilon, \varepsilon^{2}, \ldots\right\} \subseteq \operatorname{Supp}(u)$ or $\left\{l, \varepsilon^{-1}, \varepsilon^{-1}, \varepsilon^{-2}, \ldots\right\} \subseteq \operatorname{Supp}(u)$.

Proof. If $\varepsilon=\imath$, then $u=1 / 2$ and $\left\{l, \varepsilon, \varepsilon^{2}, \ldots\right\}=\left\{l, \varepsilon^{-1}, \varepsilon^{-2}, \ldots\right\}=$ $\{l\}=\operatorname{Supp}(u)$. Suppose $\varepsilon \neq t$. Since $1=u+u \bar{\varepsilon}, 0 \neq 1_{\imath}=u_{t}+u_{\varepsilon^{-1}}$ and hence $u_{i} \neq 0$ or $u_{\varepsilon-1} \neq 0$. If $u_{i} \neq 0$, then, since $0=1_{\varepsilon}=u_{\varepsilon}+$ $u_{1}, u_{\varepsilon} \neq 0$. Repeating this proof $n$ times shows that $u_{\varepsilon^{n}} \neq 0$, and hence that $\left\{l, \varepsilon, \varepsilon^{2}, \ldots\right\} \subseteq \operatorname{Supp}(u)$. On the other hand, if $u_{\varepsilon-1} \neq 0$, then, since $0=1_{\varepsilon^{-1}}=u_{\varepsilon^{-1}}+u_{\varepsilon^{-2}}, u_{\varepsilon^{-2}} \neq 0$. In this case, repeating the proof $n$ times shows that $u_{\varepsilon^{-n}} \neq 0$, and hence that $\left\{l, \varepsilon^{-1}, \varepsilon^{-2}, \ldots\right\} \subseteq \operatorname{Supp}(u)$.

Proposition 4.4. Let $T$ be a totally ordered field, let $\left\{t_{\gamma, \delta}\right\} \subseteq T$ be a factor set, let $(\Delta, \cdot, \geq)$ be a rooted abelian group, and let $X$ be a supporting 
subset of $2^{\Delta}$. If $\left({ }_{X} \prod_{\Delta} T,\left\{t_{\gamma, \delta}\right\},+, \cdot, \geq\right)$ is a field and $\Phi \subseteq \Delta$ has at most two elements, then there exists $\varphi \in \Phi$ such that $\bigcup_{n=1}^{\infty}\left(\varphi^{-1} \Phi\right)^{n} \in X$.

Proof. If $\Phi$ has one element, then $\bigcup_{n=1}^{\infty}\left(\varphi^{-1} \Phi\right)^{n}=\{l\} \in X$. If $\Phi=$ $\{\gamma, \eta\}$ and $\varepsilon=\gamma \eta^{-1}$, then $\bigcup_{n=1}^{\infty}\left(\varphi^{-1} \Phi\right)^{n}=\left\{l, \varepsilon, \varepsilon^{2}, \ldots\right\}$ or $\bigcup_{n=1}^{\infty}\left(\varphi^{-1} \Phi\right)^{n}$ $=\left\{l, \varepsilon^{-1}, \varepsilon^{-2}, \ldots\right\}$. Since ${ }_{x} \prod_{\Delta} T$ is a field, there exists by Lemma 4.3 $u \in{ }_{X} \prod_{\Delta} T$ such that either $\left\{l, \varepsilon, \varepsilon^{2}, \ldots\right\} \subseteq \operatorname{Supp}(u)$ or $\left\{l, \varepsilon^{-1}, \varepsilon^{-2}, \ldots\right\}$ $\subseteq \operatorname{Supp}(u)$. Proposition 4.4 then follows immediately from $\left(P_{3}\right)$.

Corollary 4.5. Let $L$ be a lattice-ordered field in which $1>0$, and suppose that $L$ satisfies the following conditions: the basic elements of $L$ are totally positive; there exists a basis $B$ of $L$ such that $b^{\perp \perp}$ is unbounded for all $b \in B$; and for all positive elements $u, v$ of $L, B(u v) \subseteq(B(u) B(v))^{\perp \perp}$ and $\left\{(a, c) \in B(u) \times B(v) \mid(a c)^{\perp \perp}=1^{\perp \perp}\right\}$ is finite. If $F \subseteq B$ has at most two elements, then there exists $v \in L$ and $m \in F$ such that $\bigcup_{n=1}^{\infty}\left(m^{-1} F\right)^{n} \subseteq$ $B(v)$.

Proof. The corollary follows from Proposition 4.4 and [19, Theorem 7.1] and its proof.

Corollary 4.5 indicates that the final hypothesis of Proposition 4.2 is in general too strong, if not redundant. If $L$ is algebraic over $M(L)$, then our final result shows that we can dispense altogether with this hypothesis and several others as well. In fact, the equivalence of (i) and (ii) below shows that the structure theorem for lattice-ordered groups [5, page 3.27] carries over to lattice-ordered fields with only one additional hypothesis, namely, that the basic elements be totally positive. Note that if $L$ is finite-dimensional over $M(L)$, then it is certainly algebraic over $M(L)$, and hence the following result generalizes [19, Theorem 10.1]. Recall that a set of positive elements $\left\{g_{\alpha}\right\}$ of a lattice-ordered group $G$ is disjoint if $0=g_{\alpha} \wedge g_{\beta}$ whenever $\alpha \neq \beta$.

Proposition 4.6. For a lattice-ordered field $L$ in which $1>0$, the following statements are equivalent.

(i) There exist a trivially ordered torsion abelian group $(\Delta, \cdot, \geq)$, a factor set $\left\{m_{\alpha, \beta}\right\} \subseteq M(L)$, and an isomorphism $\lambda: L \rightarrow\left(\sum_{\Delta} M(L)\right.$, $\left.\left\{m_{\alpha, \beta}\right\},+, \cdot \geq\right)$ of lattice-ordered fields.

(ii) The basic elements of $L$ are totally positive; no convex totally ordered subgroup of $L$ is bounded; and each positive element of $L$ exceeds at most a finite number of disjoint elements. 
(iii) The basic elements of $L$ are totally positive; $L$ has a basis $B$; for all positive elements $u, v$ of $L,\left\{(a, c) \in B(u) \times B(v) \mid(a c)^{\perp \perp}=1^{\perp \perp}\right\}$ is finite; and $L$ is algebraic over $M(L)$.

Proof. By Propositions 3.1, 3.2 and 4.1, (i) implies (iii).

(iii) $\Rightarrow$ (ii) . By hypothesis, the basic elements of $L$ are totally positive; by [19, Proposition 4.4], no convex totally ordered subgroup of $L$ is bounded. To see that each positive positive element of $L$ exceeds at most a finite number of disjoint elements, we make the following observations.

(1) If $b \in B$, then there exists $n$ such that $B_{k} b^{n} \in M(L)$. For since $b$ is algebraic over $M(L)$, there exist $\beta_{i} \in M(L)$ such that $\beta_{k} b^{k}+\beta_{k-1} b^{k-1}+$ $\cdots+\beta_{1} b+\beta_{0}=0$. Then $\beta_{k} b^{k}+\beta_{k-1} b^{k-1}+\cdots+\beta_{1} b=-\beta_{0} \in M(L)$ and hence $\beta_{n} b^{n} \in M(L)$ for some $n$. It follows that $b^{n} \in M(L)$.

(2) If $1<u \in L$ and $i>0$, then $B\left(u^{i}\right) \supseteq B\left(u^{i-1}\right)$. For if $B \in B(L)$ is such that $b<u^{i-1}$, then $b<b u<u^{i}$.

(3) If $1<u \in L$, then there exists $n>0$ such that for all $i \geq n$, $B\left(u^{i}\right)=B\left(u^{n-1}\right)$. For since $u$ is algebraic over $M(L)$, there exist $n>0$ such that for all $i \geq n$, there exist $v_{1}, \ldots, v_{i} \in M(L)$ such that $u^{i}+$ $v_{i-1} u^{i-1}+\cdots+v_{1} u+v_{0}=0$. Then $u^{i}=-v_{i-1} u^{i-1}-\cdots-v_{0}$, and hence by (2), $B\left(u^{i}\right) \subseteq \bigcup_{k=0}^{i-1} B\left(u^{k}\right)=B\left(u^{i-1}\right)$. It follows that $B\left(u^{i}\right) \subseteq B\left(u^{i-1}\right) \subseteq \cdots \subseteq$ $B\left(u^{n-1}\right)$. By $(2)$, we must have $B\left(u^{i}\right)=B\left(u^{i-1}\right)=\cdots=B\left(u^{n-1}\right)$.

(4) If $1<u \in L$, then there exists $n>0$ such that $\left\{b^{i} \mid b \in B(u)\right.$, $i>0\} \subseteq B\left(u^{n}\right)^{\perp \perp}$. If $b \in B(L)$ is such that $b<u$ and $i>0$, then $b^{i}<u^{i}$, and hence $\left\{b^{i} \mid b \in B(u), i>0\right\} \subseteq\left[\bigcup_{i=1}^{\infty} B\left(u^{i}\right)\right]^{\perp \perp}$. Observation (4) then follows from (3) above.

Now suppose by way of contradiction that $0<v \in L$ exceeds an infinite number of disjoint elements. Then $u=v+1$ also exceeds an infinite number of disjoint elements and hence $B(u)$ is infinite. Then by (1),

$$
\left\{(c, d) \in B(u) \times\left[\left\{b^{i} \mid b \in B(u), t>0\right\}^{\perp \perp} \cap B\right] \mid c d \in 1^{\perp \perp}\right\}
$$

is infinite, and hence by (4) there exists $n>0$ such that $\{(c, d) \in B(u) \times$ $\left.B\left(u^{n}\right) \mid c d \in 1^{\perp \perp}\right\}$ is infinite. This contradicts the third hypothesis of (iii), and hence we conclude that every positive element of $L$ exceeds at most a finite number of disjoint elements.

(ii) $\Rightarrow$ (i). By hypothesis, the basic elements of $L$ are totally positive, and by [5, page 3.27, Proposition], every positive element of $L$ is the join of a finite number of basic elements. The remaining three conditions of Propositions 4.1 (ii) follow from this observation, and hence by Proposition 4.1 , there exist a rooted abelian group $(\Delta, \cdot, \geq)$, a supporting subset $X$ of 
$2^{\Delta}$, a factor set $\left\{m_{\alpha, \beta}\right\} \subseteq M(L)$, and a one-to-one homomorphism $\lambda: L \rightarrow$ $\left({ }_{X} \prod_{\Delta} M(L),\left\{m_{\alpha, \beta}\right\},+, \cdot, \geq\right)$ of lattice-ordered algebras over $M(L)$ such that $\lambda(L)$ contains $\sum_{\Delta} M(L)$. Since every positive element of $L$ is the join of only a finite number of basic elements, in fact, $\lambda(L)=\sum_{\Delta} M(L)$. If $\varepsilon \in \Delta$, then $u=(1+\bar{\varepsilon})^{-1}$ exists in $\sum_{\Delta} M(L)$, and hence $\operatorname{Supp}(u)$ must be finite. But by Lemma 4.3 , either $\left\{l, \varepsilon, \varepsilon^{2}, \ldots\right\} \subseteq \operatorname{Supp}(u)$ or $\left\{l, \varepsilon^{-1}, \varepsilon^{-2}, \ldots\right\} \subseteq \operatorname{Supp}(u)$. It follows that $\varepsilon$ must be torsion. It is wellknown (cf. [8, page 15]) and easy to see that every torsion partially ordered group is trivially ordered.

We conclude by noting that the power series fields whose constructions are described above give many examples of lattice-ordered fields which satisfy the hypotheses of Proposition 4.2. There are many other examples of latticeordered fields whose basic elements are not totally positive (cf. [19, Example 10.3]). These observations lead to the question whether there are latticeordered fields whose basic elements are totally positive but which fail to satisfy one or more of the other hypotheses of Propositions 4.2. I have been unable to find such examples. In particular, I have not found an example of a lattice-ordered field which fails to satisfy the condition on countable subsets of sets of the form $B(u)$ but does satisfy the other hypotheses of Proposition 4.2. As was indicated above, Corollary 4.5 seems to suggest that such an example may be difficult, if not impossible, to find.

\section{References}

[1] M. Anderson, T. Feil, Lattice-ordered Groups: An Introduction (D. Reidel (Kluwer), Dordrecht, Holland, 1987).

[2] A Bigard, K. Keimel, S. Wolfenstein, Groupes et Anneaux Réticulés (Lecture Notes in Math. 608, Springer-Verlag, Berlin, FRG, 1977).

[3] A. H. Clifford, 'Partially ordered abelian groups', Ann. Math. 41 (1940), 465-473.

[4] P. Conrad, 'Generalized semigroup rings', J. Indian Math. Soc. 21 (1957), 73-95.

[5] P. Conrad, Lattice-ordered Groups (Tulane University, New Orleans, USA, 1970).

[6] P. Conrad, J. Dauns, 'An embedding theorem for lattice-ordered fields', Pacific J. Math. 30 (1969), 385-398.

[7] P. Conrad, J. Harvey, W. C. Holland, 'The Hahn embedding theorem for lattice-ordered groups', Trans. Amer. Math. Soc. 108 (1963), 143-169.

[8] L. Fuchs, Partially Ordered Algebraic Structures (Pergamon Press, Oxford, UK 1963).

[9] H. Hahn, 'Über die nichtarchimedischen Grössensysteme', Sitzungsber. Kaiserlichen Akad. Wiss. Vienna, Math. Natur. Kl. Abt. IIa 116 (1907), 601-653.

[10] N. Jacobson, Lectures in Abstract Algebra III: Theory of Fields and Galois Theory (D. van Nostrand, Princeton, USA, 1964).

[11] I. Kaplansky, 'Maximal fields with valuations', Duke Math. J. 9 (1942), 303-321. 
[12] P. Lorenzen, 'Abstrakte Begründung der multiplikativen Idealtheori' Math. Z. 45 (1939), 533-553.

[13] B. H. Neumann, 'On ordered division rings', Trans. Amer. Math. Soc. 66 (1949), 202252.

[14] R. H. Redfield, 'Algebraic extensions and lattice-ordered fields', Analysis Paper 15, Monash University, Melbourne, 1975.

[15] R. H. Redfield, ' $O$-subfields of 1-fields', Abstract 75T-A225, Notices Math. Soc. 22 (1975), A-618.

[16] R. H. Redfield, 'Banaschewski functions and rings embeddings', Ordered Algebraic Structures, Proc. Conf. Curaçao, 1988; pp. 247-255 (Kluwer Academic Publishers, Dordrecht, 1989).

[17] R. H. Redfield, 'Representations of rings via Banaschewski functions' (in preparation).

[18] R. H. Redfield, 'Constructing lattice-ordered fields and division rings', Bull. Austral. Math. Soc. 40 (1989), 365-369.

[19] R. H. Redfield, 'Lattice-ordered fields as convolution algebras', J. of Algebra (to appear).

[20] P. Ribenboim, 'Rings of generalized power series: nilpotent elements', preprint.

[21] P. Ribenboim, 'Rings of generalized power series II: units, zero divisors and semisimplicity', preprint.

[22] P. Ribenboim, 'Noetherian rings of generalized power series', preprint.

[23] J. J. Rotman, The Theory of Groups: An Introduction (Allyn and Bacon, Boston, USA 1965).

[24] N. Schwartz, 'Lattice-ordered fields', Order 3 (1986), 179-194.

Department of Mathematics and Computer Science

Hamilton College, Clinton

New York 13323

USA 